\title{
Experiencia de un equipo médico de emergencia del IMSS durante el sismo en México en setiembre de 2017
}

\author{
Felipe Cruz-Vega, ${ }^{1}$ Jorge Loría-Castellanos, ${ }^{1}$ Juan Carlos Sánchez-Echeverría ${ }^{1}$ \\ y Rodolfo Sosa-Barragán²
}

Forma de citar

Cruz-Vega F, Loría-Castellanos J, Sánchez-Echeverría JC, Sosa-Barragán R. Experiencia de un equipo médico de emergencia del IMSS durante el sismo en México. Rev Panam Salud Publica. 2018;42:e79. https://doi.org/10.26633/RPSP.2018.79

RESUMEN El objetivo de este trabajo es describir las acciones desarrolladas por el equipo médico de emergencia (EMT por sus siglas en inglés) del Instituto Mexicano del Seguro Social (IMSS) durante la emergencia derivada del sismo del 7 de setiembre de 2017, el cual tuvo una magnitud de 8,2 grados con epicentro a $133 \mathrm{~km}$ al suroeste de Pijijiapan, Chiapas, México. Una vez realizada la evaluación inicial intersectorial de la emergencia, se activó el sector salud. Dentro de esta respuesta, y una vez determinado un número elevado de lesionados y un riesgo alto para la salud pública de la población afectada, el IMSS desplegó en la región un EMT capacitado y entrenado para apoyar en la respuesta de atención médica y quirúrgica de lesionados. Las acciones del EMT, conformado por especialistas en urgencias médico-quirúrgicas y desastres, incluyeron la atención médica de 252 pacientes, la gestión del traslado de 57 pacientes y la colaboración en la habilitación de un hospital provisorio. Para mejorar la respuesta en situaciones de desastres, se requiere seguir el proceso de integración, registro y capacitación de los EMT a nivel nacional e internacional.

Palabras clave Atención de emergencias; desastres; sismo; México.

El objetivo de este trabajo es describir las acciones desarrolladas por el equipo médico de emergencia del Instituto Mexicano del Seguro Social (IMMS) durante la emergencia derivada del sismo del 7 de setiembre de 2017.

El análisis Respuesta de salud al terremoto de Haití, elaborado por la Organización Panamericana de la Salud

División de Proyectos Especiales en Salud, Dirección de Prestaciones Médicas, Instituto Mexicano del Seguro Social, México. Enviar correspondencia a Jorge Loría-Castellanos, jloriac@hotmail.com

2 Hospital Regional 25, Instituto Mexicano del Seguro Social, México.
(OPS) en 2012, demostró que, de forma similar a otros desastres, aunque se contó con gran cantidad de equipos médicos eficientes, también hubo un importante número de respondientes que no estaban preparados o incluso llegaron a ser incompetentes. Este estudio evidenció la necesidad de desarrollar principios, criterios y estándares para la respuesta de los equipos médicos en casos de emergencias y desastres, en línea con los procesos globales para mejorar las normas y estándares humanitarios (1-3).

En este sentido, la OPS estableció el Plan de acción para coordinar la asistencia humanitaria en las Américas, aprobado en el 53. ${ }^{\circ}$ Consejo Directivo de la OPS, en el que se considera la implementación de procedimientos de respuesta y los mecanismos nacionales flexibles de registro para los equipos médicos de emergencia. En el 55. ${ }^{\circ}$ Consejo Directivo se aprobó el Plan de acción para la reducción del riesgo de desastres 2016-2021, en el que se insta a fortalecer, a nivel nacional, el desarrollo y la actualización de conocimientos y procedimientos de los equipos de respuesta a desastres y emergencias (4).

Los equipos médicos de emergencias (EMT por sus siglas en inglés) son grupos

Este es un artículo de acceso abierto distribuido bajo los términos de la licencia Creative Commons Attribution-NonCommercial-NoDerivs 3.0 IGO, que permite su uso, distribución y reproducción en cualquier medio, siempre que el trabajo original se cite de la manera adecuada. No se permiten modificaciones a los artículos ni su uso comercial. Al reproducir un artículo no debe haber ningún indicio de que la OPS o el artículo avalan a una organización o un producto específico. El uso del logo de la OPS no está permitido. Esta leyenda debe conservarse, junto con la URL original del artículo. 
de profesionales de la salud (médico, enfermería, fisioterapeutas, paramédicos, entre otros) que proveen tanto cuidado clínico directo a poblaciones afectadas por emergencias y desastres como apoyo a sistemas locales de salud. Los EMT pueden ser gubernamentales (civiles y militares) o estar bajo la esfera de organizaciones no gubernamentales; su respuesta puede ser nacional o internacional; pueden estar enfocados al manejo de patologías médicas, traumatológicas, quirúrgicas y epidemiológicas, entre otros y el objetivo es reducir la pérdida de vidas, mejorar la recuperación de la salud y la prevención de discapacidades en el largo plazo en las emergencias y los desastres (5).

De acuerdo a sus características de personal, recursos, infraestructura y funciones, los EMT se clasifican en tres niveles (5):

- Atención ambulatoria de emergencia (nivel 1): tiene la capacidad de otorgar cuidado inicial de emergencia a pacientes ambulatorios. Realiza acciones de triaje, evaluación, primeros auxilios, estabilización y transferencia de pacientes con traumatismo grave y otras emergencias, además de cuidados definitivos en pacientes con traumatismos menor y otras urgencias. Debe contar con equipo ligero, portátil y adaptable con los recursos para atender diariamente a 50 pacientes (móvil) o 100 pacientes (fijo).

- Atención quirúrgica de emergencia de nivel hospitalario (nivel 2): tiene la capacidad para realizar cuidados agudos hospitalarios, cirugía general, obstetricia y traumatológica. Realiza acciones de triaje quirúrgico, evaluación, manejo básico de fracturas y definitivo de heridas y cirugía de control daños, general y obstétrica de emergencia. Cuenta con equipo para atención hospitalaria, anestesia básica, transfusiones, radiología, laboratorio, rehabilitación, ambiente quirúrgico limpio, un área quirúrgica con un quirófano y veinte camas para hospitalización. Tiene capacidad para realizar siete operaciones mayores o 15 operaciones menores por día.

- Atención hospitalaria de referencia (nivel 3): tiene la capacidad para otorgar atención quirúrgica de referencia para pacientes hospitalizados con patologías complejas, incluida la capacidad de cuidados intensivos. Realiza, además de las acciones del nivel 2, acciones de atención ortopédica y reconstrucción de heridas complejas. Cuenta con servicios de radiología, ultrasonido, transfusiones, laboratorio, rehabilitación, anestesia pediátrica y de adultos, terapia intensiva, ventiladores y ambiente quirúrgico estéril, así como un área quirúrgica con dos quirófanos, 40 camas para hospitalización y de cuatro a seis camas de terapia intensiva. Tiene capacidad para realizar 15 operaciones mayores o 30 operaciones menores por día.

El IMMS es el mayor proveedor del sistema de salud de México y uno de los más grandes de América Latina; a través de la División de Proyectos Especiales en Salud coordina la respuesta médica ante diferentes contingencias que se presentan en el país y el extranjero. Sus equipos han participado en desastres como las inundaciones del estado de Tabasco, México (2007), así como en la misión de ayuda enviada en el terremoto de Haití de 2010 (6-7).

El presente artículo describe la experiencia del EMT del IMSS durante las afectaciones por el sismo en el estado de Oaxaca, México, en 2017.

\section{EVENTO}

Casi treinta y dos años después de los catastróficos eventos sísmicos de setiembre de 1985 en la Ciudad de México, el día 7 de setiembre de 2017, a las 23:49:18 h (tiempo del Centro de México) se presentó un sismo de magnitud 8,2 de la escala de Richter con referencia de localización del epicentro a $133 \mathrm{~km}$ al suroeste de Pijijiapan, Chiapas, en latitud: $14.85^{\circ}$ y longitud: $-94.11^{\circ}$ a una profundidad de $58 \mathrm{~km}$. Hasta el día en que se escribe este documento (29 de setiembre de 2017), ha presentado 5612 réplicas, las dos mayores de magnitud 6,1. El mecanismo focal del sismo mostró una falla de tipo normal, la cual es característica de un sismo intraplaca. En esta región, la placa de Cocos subduce por debajo de la placa de América del Norte (8).

En Oaxaca, 41 municipios resultaron afectados, entre ellos, Juchitán, Matías Romero, Unión Hidalgo y San Dionisio del Mar; también hubo afectaciones en los estados de Chiapas y Tabasco. El sismo causó la muerte de 96 personas en diferentes municipios del país, en su mayoría en Juchitán, Oaxaca (76 personas). Aunque de las instalaciones del IMSS solo la Unidad de Medicina Familiar 06 en Juchitán y el Hospital Rural de Matías Romero tuvieron afectaciones no estructurales, conservaron su funcionalidad; no obstante, las afectaciones de la Secretaría de Salud incluyeron al Hospital General "Doctor Aurelio Valdivieso", el Hospital General de Ixtepec y el Hospital General Juchitán. Fue necesario reubicar a más de 100 pacientes y se vio comprometida la respuesta en materia de salud de la zona (9).

\section{ACCIONES DEL EMT DEL IMSS}

Una vez activada la emergencia, el Comité Nacional de Emergencias determinó la necesidad de enviar apoyo médico a las zonas afectadas. Como parte del Plan de Contingencia Nacional, el IMSS gestionó el envío de su EMT a Juchitán, Oaxaca, como apoyo a la respuesta del sector salud.

El EMT laboró en la zona desde el 8 hasta el 14 de setiembre de 2017 y estuvo conformado por cuatro especialistas en urgencias médico quirúrgicas y desastres y cuatro enfermeras especialistas en urgencias y terapia intensiva.

Este grupo contaba con equipo para respuesta inmediata de urgencias, que incluía un monitor-desfibrilador, material de curación, instrumental y medicamentos para reanimación básica y avanzada, entro otros. Como célula especializada complementaria, se integró un equipo quirúrgico conformado por un cirujano general, un ortopedista, un anestesiólogo y una enfermera quirúrgica. Se movilizó también una unidad quirúrgica móvil, la cual cuenta con autonomía para realizar intervenciones quirúrgicas, ortopédicas y ginecoobstétricas de urgencia.

La coordinación en campo se realizó en forma directa con las autoridades de la Secretaría de Gobernación, la Secretaría de Salud Federal y la Secretaría de Salud Estatal; la coordinación con las autoridades locales y centrales del IMSS se realizó a través del Centro Virtual de Operaciones en Emergencias y Desastres (CVOED) (10-11).

Entre las acciones realizadas, se encuentran:

- Atención médica de 252 pacientes, incluidos tres partos. Se desarrollaron actividades de triaje, evaluación, primeros auxilios, estabilización y transferencia de pacientes con traumatismo grave y otras emergencias no traumatológicas, así como cuidados 
definitivos para pacientes con traumatismo menor y otras emergencias no traumatológicas.

- Gestión del traslado de 57 pacientes, dos de ellos de alta complejidad por tratarse de pacientes de ginecoobstetricia (una paciente con embarazo de 34 semanas con trombocitopenia grave y una paciente posoperada de cesárea por eclampsia).

- Evaluación estructural y funcionalidad de las unidades de salud (IMSS y Secretaría de Salud) en Juchitán, Ixtepec, Salina Cruz, Ixtlaltepec y Matías Romero.

- Habilitación de la instalación de un hospital provisional en las inmediaciones del Instituto de Estudios Superiores del Istmo de Tehuantepec (IESIT), en Juchitán.

- Colaboración con instituciones federales y estatales en la instalación, en el Instituto de estudios Superiores del Istmo de Tehuantepec, de las áreas de triaje, urgencias, reanimación, hospitalización (medicina interna, cirugía, pediatría, ginecología y traumatología), expulsión, recuperación, central de equipo y esterilización, farmacia, bodega, ropería, salas de espera, comedor, etc.)

- Coordinación en la instalación de la unidad quirúrgica móvil.

- Equipar carros rojos o de urgencias médicas para tener a disposición.

- Preparación de equipos de cirugía.

- Habilitación de área de cadáveres.

- Coordinación traslados terrestres y aéreos.

- Presentación de las instalaciones habilitadas a las autoridades federales y estatales.

\section{DISCUSIÓN}

La iniciativa EMT, como parte del programa Global Health Emergency Workforce de la Organización Panamericana de la Salud/Organización Mundial de la Salud (OPS/OMS), pretende superar la tan criticada falta de preparación y coordinación que se presenta en la ayuda en zonas de desastres.

Un componente esencial para alcanzar este objetivo es el nivel adecuado de educación y entrenamiento; en este sentido, esta iniciativa propone un marco de aprendizaje operacional de tres pasos:

1. Asegurar las competencias profesionales (certificación) del personal profesional competencia y licencia para practicar.

2. Apoyar la adaptación de capacidades profesionales técnicas y no técnicas en el contexto de bajos recursos y emergencia.

3. Prepararse para un rendimiento efectivo del equipo en el campo (12).

Gracias a la iniciativa de los EMT, los gobiernos y las personas afectadas por emergencias pueden contar con atención médica inmediata, accesible en la zona afectada, la respuesta rápida y previsible, brindada por equipos médicos capacitados, con entrenamiento especializado y autosuficientes.

La misión de los EMT es la reducción de la pérdida de vidas, la recuperación de la salud y la prevención de discapacidades en el largo plazo en las emergencias y los desastres, a través del despliegue rápido y coordinado de personal equipado, con calidad asegurada.

Entre los pasos iniciales para esta acción se encuentra el contar con un censo confiable de personal de la salud que se involucra en la respuesta nacional, internacional o ambas en casos de emergencia o desastre. En este sentido, el IMSS cuenta desde 2015 con un censo actualizado de personal médico y de enfermería disponible en todos los estados del país, lo que facilitó que en las primeras horas del evento se movilizaran e iniciaran sus actividades. Además, estos equipos médicos han recibido capacitación en atención psicológica para primeros respondedores, en atención a saldo masivo de víctimas y respuesta en emergencias por agentes químicos, biológicos, radiológicos, nucleares y explosivos (QBRNE). Un punto pendiente sería generar un proceso continuo de registro nacional e interinstitucional en donde se consideren no solo los aspectos laborales del personal, sino garantizar su capacitación continua.

La conformación de los EMT se rigió

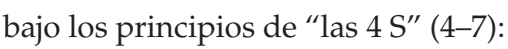

- Personal (staff). Conformado por especialistas en urgencias con capacitación en desastres. Su registro previo optimizó los tiempos y facilitó su despliegue inmediato a las zonas siniestradas.

- Autosuficiencia de material (stuff). El EMT contaba con autonomía y autosuficiencia de material, medicamentos, recursos, alimentación y hospedaje.
- Espacio necesario (space). Quizás este fue uno de los puntos débiles ya que, aunque se contó con el apoyo de la unidad móvil, hizo falta contar con unidades de despliegue.

- Sistema de gestión (system). Se organizaron las actividades, funciones y líneas de mando junto a las autoridades locales y nacionales durante todo el despliegue.

La respuesta de este EMT se coordinó con las autoridades de nivel central a través del CVOED, símil institucional de la Cëlula de Información y Cooridnación Médica (CICOM) que propone la OPS, lo que facilitó no solo el manejo de información, sino también la gestión de recursos $(5,7)$.

Tan solo unos días después del sismo, el 19 de setiembre, se vivieron otra vez las afectaciones de un sismo, en esa ocasión en la Ciudad de México. La respuesta del EMT fue inmediata y su experiencia en este evento aún está en desarrollo.

Los puntos clave para la adecuada implementación y funcionamiento de los EMT son la información y la capacitación. Al respecto, la Secretaría de Equipos Médicos de Emergencia de la OMS, junto con la OPS, han organizaron diferentes Reuniones Globales (como en Panamá en 2015 y en México en 2017) en las que los participantes abordan temas sobre actualizaciones de la iniciativa global EMT y la reforma de la OMS para la respuesta en brotes y emergencias.

Resulta crucial también continuar con el proceso de verificación y registro de los EMT tanto a nivel nacional como internacional, proceso en el que al momento solo unos pocos países en el mundo, como Costa Rica e Israel, han mostrado avances significativos. De esta manera, "se asegura que equipos médicos internacionales que prestan atención clínica dentro de los estándares mínimos de calidad puedan ser desplegados a tiempo, siendo fundamental para ayudar a salvar vidas y evitar discapacidades, así como proteger la salud de las poblaciones afectadas por una emergencia" (13).

\section{CONCLUSIÓN}

En este artículo se trata el primer acercamiento al funcionamiento de un EMT en México. Este cumplió con el indicador de productividad para EMT móvil (50 pacientes/día), aunado a las actividades de coordinación y logística de las instalaciones. 
Se detectaron las siguientes áreas de oportunidad: la necesidad de contar con los insumos para ser un EMT de nivel 2 fijo, a fin de contar con equipamiento que proporcione mayor autonomía, con más capacidad de despliegue en el área. Esto se relaciona con la complejidad de traslado de pacientes, no solo por la gravedad de sus lesiones, sino también por la limitada infraestructura hospitalaria instalada en la región, lo que deriva en traslados con distancias mayores.

\section{RECOMENDACIONES}

Es necesario un proyecto nacional para el diseño, creación y registro de Equipos Médicos de Emergencia, así como su capacitación, asesoría y coordinación a fin de desarrollar procedimientos y herramientas específicas. Es primordial la participación en foros regionales y globales para el intercambio de conocimiento y buenas prácticas, así como generar líneas de trabajo internacionales.

\section{REFERENCIAS}

1. Organización Panamericana de la Salud (OPS). La respuesta de salud al terremoto de Haití en enero de 2010. Washington, D.C.: OPS; 2012. Disponible en: http://www. paho.org/disasters/index.php?option= com_content\&view $=$ category\&id=1216\&layout=blog\&Itemid=1136\&lang=es

2. Lind K, Gerdin M, Wladis A, Westman L, von Schreeb J. Time for order in chaos! A health system framework for foreign medical teams in earthquakes. Prehosp Disaster Med. 2012;27(1):90-3.

3. Van Hoving DJ, Wallis LA, Docrat F, De Vries S. Haiti disaster tourism: a medical shame. Prehosp Disaster Med. 2010;25(3): 201-2.

4. Organización Panamericana de la Salud. Plan estratégico de la Organización Panamericana de la Salud 2014-2019. Disponible en: http://iris.paho.org/xmlui/handle/ 123456789/4034

5. Organización Panamericana de la Salud. Nota técnica sobre los requisitos mínimos de los Equipos Médicos de Emergencia (EMT) que responden a desastres y emergencias en las Américas. Principios y estándares para la clasificación y registro de los EMT. Disponible en: http://www. paho.org $/$ disasters $/$ index.php?option= com_docman\&task=doc_download\&gid= 2411\&Itemid=\&lang=es

6. Loría-Castellanos J, Cruz-Vega F, GomezZárate E. Experience of IMSS medical equipment in rescue efforts in Haiti. Prehosp Disaster Med. 2011;26 (S1):104.

7. División de Proyectos Especiales en Salud. Instituto Mexicano del Seguro Social. Plan Institucional Frente a Emergencias y Desastres. Marco de Actuación General. Disponible en: http:/ /cvoed.imss.gob.mx/ COED/home/normativos/DPM/archivos/PIFED.pdf

8. Grupo de trabajo del Servicio Sismológico Nacional, UNAM. Sismo de Tehuantepec (2017-09-07 23:49 M 8.2). Reporte especial. Disponible en: http:/ /www.ssn.unam.mx/ sismicidad / reportes-especiales / 2017 / SSNMX_rep_esp_20170907_Tehuantepec_ M82.pdf

9. Secretaría de Gobernación de México. Más de dos mil trabajadores de salud en zona afectada por sismo en Oaxaca. Consultado en: https://www.gob.mx/salud/prensa/ 374-mas-de-dos-mil-trabajadores-desalud-en-zona-afectada-por-sismo-enoaxaca?idiom $=$ es

10. Cruz-Vega F, Saavedra-Gómez JL, JimenezSánchez K, Elizondo-Argueta S, LoríaCastellanos J. Centro Virtual de Operaciones en Emergencias y Desastres (CVOED). Sistema informático en línea que ayuda a las instituciones para enfrentar situaciones de emergencia o desastres. Arch Med Urg Mex. 2013;5(2):51-2.

11. Bautista Reyes AL, Cruz Vega F, Loría Castellanos J. Uso del centro virtual de
Es importante continuar con la difusión de las experiencias en la implementación y funcionamiento de estos EMT a nivel internacional.

Conflicto de intereses. Ninguno declarado por los autores.

Declaración. Las opiniones expresadas en este manuscrito son responsabilidad del autor y no reflejan necesariamente los criterios ni la política de la RPSP/ PAJPH y/o de la OPS. operaciones en emergencias y desastres ante fenómenos perturbadores de origen natural. Arch Med Urgen Mex 2016; 8(3):90-4.

12. Amat Camacho N, Hughes A, Burkle FM, Ingrassia PL, Ragazzoni L, Redmond A, Norton I, von Schreeb J. Education and training of emergency medical teams: recommendations for a global operational learning framework. PLOS Currents Disasters. 2016(1). doi: 10.1371/currents. dis.29203368920961 1ad5e4a7a3e61520d0

13. Organización Panamericana de la Salud/ Organización Mundial de la Salud (OPS/ OMS). Equipos médicos de emergencia en Costa Rica es el primero en las Américas en ser clasificado como EM por la OMS. Disponible en: http://www.paho.org/disasters $/$ index.php?option $=$ com_content\&view $=$ article\&id=3559: equipo-medic o-de-emergencia-de-costa-rica-es-el-primero-en-las-americas-en-ser-clasificadocomo-emt-por-la-oms\&Itemid $=1178 \&$ lang $=\mathrm{es}$

Manuscrito recibido el 10 de octubre de 2017. Aceptado para su publicación, tras revisión, el 9 de marzo de 2018. 
ABSTRACT

The experience of a medical emergency team belonging to the Mexican Social

Security Institute during the earthquake in Mexico in September 2017

Keywords Ambulatory care; disasters; earthquakes; Mexico.
The objective of this article is to describe the actions undertaken by the emergency medical team (EMT) of the Mexican Social Security Institute (MSSI) during the emergency caused by the earthquake of September 7, 2017 (magnitude 8.2; epicenter 133 $\mathrm{km}$ southwest of Pijijiapan, Chiapas, Mexico). After the initial intersectoral assessment of the emergency had been conducted, the health sector stepped in. As part of this response effort, and once it was determined that a large number of people had been injured and that the health risks facing the affected population were substantial, the MSSI deployed an EMT equipped and trained to provide support in the medical and surgical treatment of injured people. The actions of the EMT, composed of specialists in medical and surgical emergencies and disaster situations, included providing medical care to 252 patients, managing the transfer of 57 patients, and helping to set up an emergency hospital. To improve response efforts during disaster situations, it is necessary to follow the process of forming, registering, and training EMTs at the national and international levels.
RESUMO

\section{Experiência de uma equipe médica de emergência do IMSS no terremoto do México em setembro de 2017}

\author{
Palavras-chave
}

O propósito deste artigo é descrever as ações empreendidas pela equipe médica de emergência (EMT) do Instituto Mexicano de Seguro Social (IMSS, instituição pública que presta serviços de saúde) na crise de emergência causada pelo terremoto de 7 de setembro de 2017, com magnitude de 8,2 graus e epicentro a $133 \mathrm{~km}$ a sudoeste de Pijijiapan, Chiapas, México. Após a avaliação intersetorial inicial de emergência, o setor da saúde foi ativado. Como parte da resposta, diante do elevado número de feridos e alto risco para a saúde pública da população afetada, o IMSS mobilizou uma EMT na região, capacitada e treinada para dar apoio à resposta de atendimento médica e cirúrgica das vítimas. A EMT, formada por especialistas em emergências médico-cirúrgicas e desastres, prestou atendimento médico a 252 pacientes, coordenou a transferência de 57 pacientes e também colaborou na preparação de um hospital provisório. A fim de melhorar a resposta a desastres, é necessário prosseguir com o processo de integração, cadastramento e capacitação das EMT ao nível nacional e internacional. 